\title{
Microbial influences on oral mucositis during radiotherapy treatment of head and neck cancer
}

\section{Anna Vesty ${ }^{1}$, Kim Gear ${ }^{2}$, Kristi Biswas ${ }^{1}$, Brett Wagner Mackenzie ${ }^{1}$, Michael W. Taylor ${ }^{3,4}$ and Richard G. Douglas ${ }^{1}$}

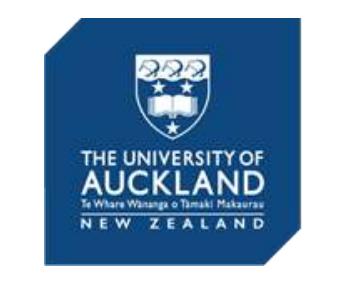

MEDICAL AND HEALTH SCIENCES
1Department of Surgery, The University of Auckland, New Zealand 2Department of Otorhinolaryngology, Auckland City Hospital, New Zealand ${ }^{3}$ School of Biological Sciences, The University of Auckland, New Zealand ${ }^{4}$ Maurice Wilkins Centre, The University of Auckland, New Zealand

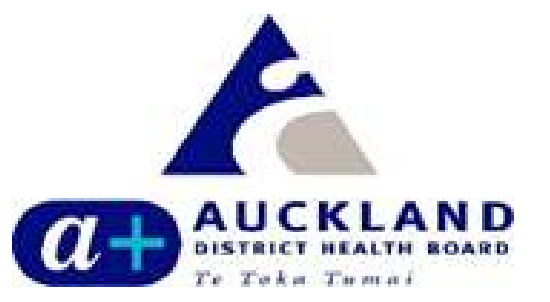

INTRODUCTION: Oral mucositis (OM) remains a significant complication developed by many patients undergoing radiotherapy $(\mathrm{RT})$ to the head and neck region.

An emerging theme suggests that Gram-negative anaerobes, including periopathogenic genera Porphyromonas and Treponema, are associated with the onset and severity of $\mathrm{OM}$ in head and neck cancer (HNC) patients ${ }^{1}$. Such observations support the hypothesis that poor oral health may leave patients more susceptible to developing $\mathrm{OM}^{2}$.

In this study, we describe changes in the oral mucosal microbiota of HNC patients during RT and correlated our findings with the incidence and severity of OM.

METHODS: Sterile swabs were used to sample sites of mucositis ulcerations in 19 patients by rolling/rubbing over the affected area. In the absence of OM, the buccal mucosa (BM) was swabbed. Patients developed OM on either the BM or lateral tongue $(\mathrm{LT})$.

Molecular microbiological techniques were use to study the bacterial communities present in the swabs. Microbial data was correlated with the onset and severity of OM, grouped as $\leq$ grade $1 \mathrm{OM}$ (no ulcerations) or $\geq$ grade $2 \mathrm{OM}$ (ulcerations present), according to the World Health Organisation Oral Mucositis Assessment Scale.

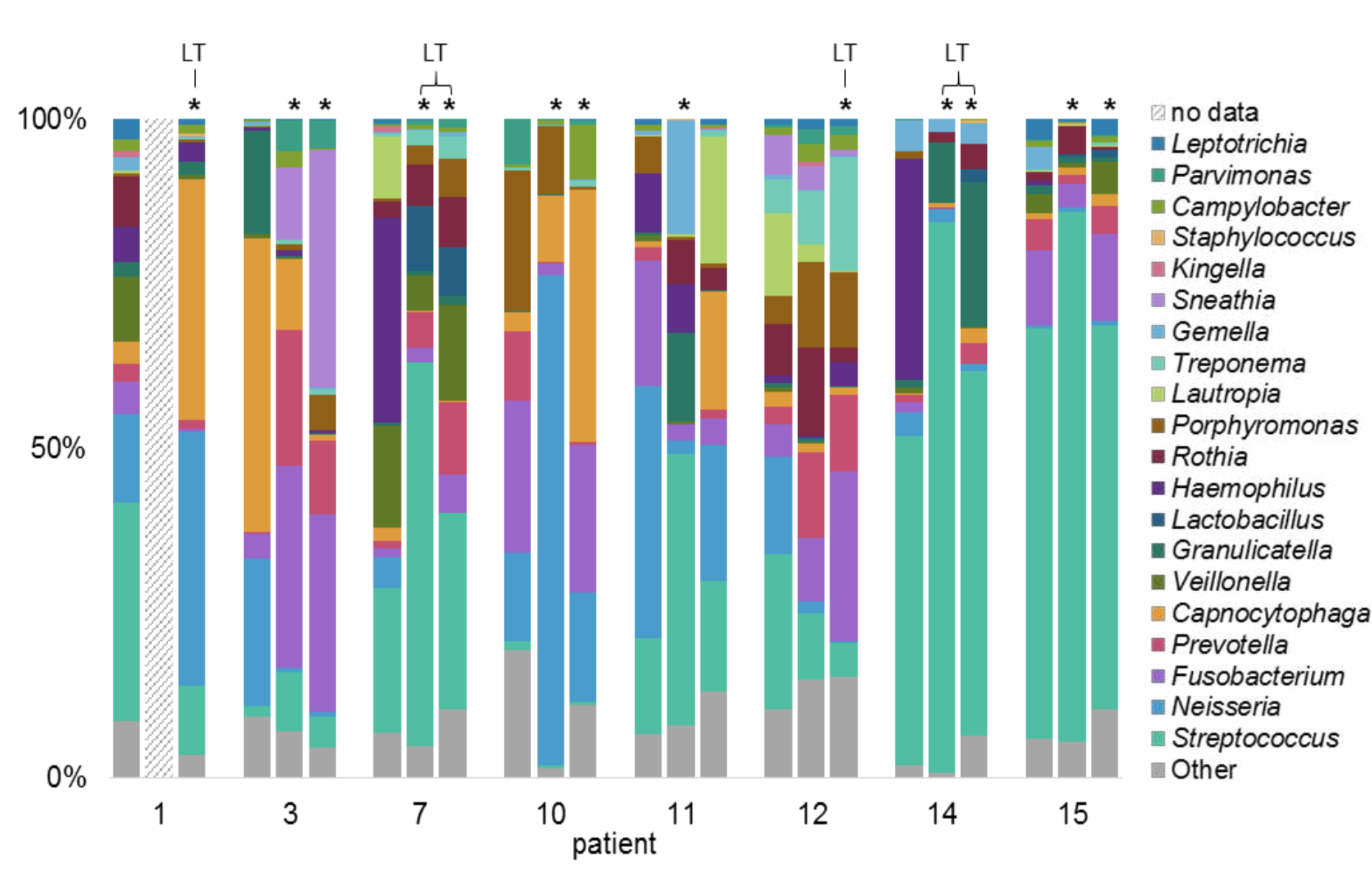

Fig 2. Swabs taken from sites of $\geq$ grade 2 OM are identified by asterisks $\left({ }^{*}\right)$; those unmarked reflect $\leq$ grade 1 swabs; LT identifies lateral tongue swabs, unlabelled bars represent BM swabs
RESULTS: The relative abundances of obligate and facultative anaerobic Gram-negative bacilli (GNB), including Bacteroidales G2, Capnocytophaga, Eikenella, Mycoplasma and Sneathia, were positively correlated with $\geq$ grade $2 \mathrm{OM}$ (Fig 1$)$.

Periopathogenic bacterial genera Porphyromonas and Tannerella were also positively correlated with mucositis ulcerations and are genera highly associated with periodontitis.

The bacterial load at sites of $\geq$ grade 2 OM was higher than at sites of $\leq$ grade $1 \mathrm{OM}$.

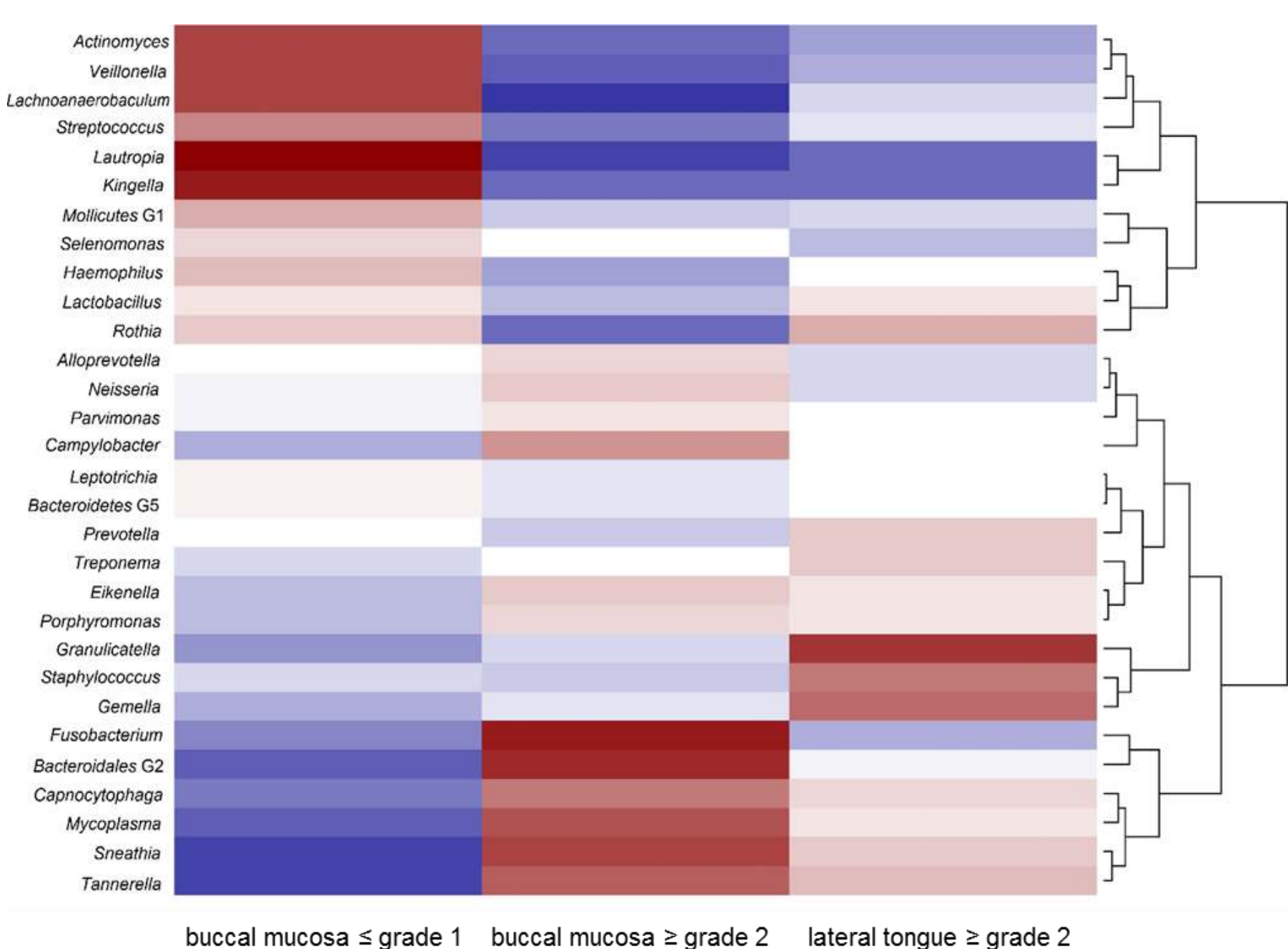

Fig 1. Heatmap visualising correlations between the 30 most abundant bacterial genera identified in swabs and the site-specific presence or absence of $\geq$ grade $2 \mathrm{OM}$. Positive correlations are visualised in shades of red, negative correlations in shades of blue

CONCLUSIONS: This study suggests anaerobic GNB, including those associated with periodontal disease, play a role in the pathogenesis of OM. Therefore, the oral bacterial community is a potential risk factor for OM. Our findings support the need for interventions aimed at minimising microbial risk factors prior to RT, for example implementing an oral hygiene protocol and the provision of oral health care advice.

${ }^{1}$ Hou J, Zheng HM, Li P, et al (2018) Distinct shifts in the oral microbiota are associated with the progression and aggravation of mucositis during radiotherapy. Radiother Oncol. https://doi.org/10.1016/j.radonc.2018.04.023

2 Khaw A, Logan R, Keefe D, Bartold M (2014) Radiation-induced oral mucositis and periodontitis - proposal for an inter-relationship. Oral Dis. https://doi.org/10.1111/odi.12199 